\title{
Medication Information Transfer: An Exploratory Study in a Rural Queensland Community
}

\author{
Amy CW Tan, Lynne M Emmerton, H Laetitia Hattingh, Victoria I Jarvis
}

\begin{abstract}
Background: Inadequate or inaccurate transfer of medication information can challenge optimal medication management. Studies of medication information transfer typically involve the hospital-community interface in urban settings. There is little research on medication information transfer between community health services, between hospitals (rural and metropolitan) and rural community settings.

Aim: To explore medication information transfer issues faced by rural healthcare providers and their perspectives on initiatives to optimise the process.

Method: Semi-structured interviews were conducted with 49 health professionals in 4 towns in rural Queensland on issues surrounding medication supply and management. Interviews were recorded, transcribed verbatim and analysed thematically. Results: Some of the issues identified included: discrepancies in medication records, lack of a coordinated system to communicate medication or prescription information between health professionals, and inadequate communication between secondary/tertiary facilities and rural primary care providers. According to the participants, the root causes of these challenges related to workforce issues and inefficiencies in communication and networking systems.

Conclusion: Key issues were identified relating to medication information transfer between healthcare providers and between healthcare settings, which impacted on optimal medication management for rural patients.
\end{abstract}

J Pharm Pract Res 2012; 42: 48-52.

\section{INTRODUCTION}

Medication management by health professionals can be challenging when changes are not communicated effectively between healthcare providers, carers and patients. ${ }^{1-6}$ Inadequate or inaccurate transfer of medication information can lead to increased hospital admissions and unplanned emergency department attendances. ${ }^{1,6,7}$ Systems that improve medication information transfer, especially at the hospital-community interface, have demonstrated error reduction. ${ }^{1,6,8}$ To achieve continuity of medication management, the Australian Pharmaceutical Advisory Council's guidelines outline that timely and effective information transfer between health services (e.g. general practice, nursing service, community pharmacy, specialist centre, hospital) is essential. ${ }^{2}$ The Public Hospital Pharmaceutical Reforms

Amy CW Tan, BPharm (Hons), PhD Candidate, Lynne M Emmerton, BPharm (Hons), PhD, MPS, Senior Lecturer, School of Pharmacy, The University of Queensland (currently Associate Professor, School of Pharmacy, Curtin University), H Laetitia Hattingh, BPharm, MPharm, PhD, GCAppLaw, Cert IV TAA, MPS, Senior Lecturer, School of Pharmacy, Griffith University, Gold Coast (currently Senior Lecturer, School of Pharmacy, Curtin University), Victoria I Jarvis, BPharm, MPS, Associate Lecturer, School of Pharmacy, The University of Queensland, St Lucia, Queensland

Address for correspondence: Amy Tan, School of Pharmacy, The University of Queensland, St Lucia Qld 4072, Australia.

E-mail: amy.tan@uqconnect.edu.au for hospitals, which have been adopted by some states aim to improve continuity between hospital and community sectors in relation to cognitive medication services and discharge processes. ${ }^{2-4}$ Ideally, public hospitals are to dispense one month's worth of discharge medications for chronic conditions under the Pharmaceutical Benefits Scheme (PBS) and hospital pharmacists dispensing these medications are to consult with patients and/or their carers. ${ }^{2,3,9-11}$ Other pharmacy services include medication reconciliation, medication information liaison (between hospital and community) and post-discharge medication reviews. ${ }^{3-8,12,13}$

Software has been developed to facilitate medication reconciliation processes in public hospitals and to communicate medication information to primary care providers. An example is the Queensland-wide electronic Liaison Medication System (eLMS), a web-based application, which facilitates medication reconciliation and produces a discharge medication record (DMR). The eLMS also feeds electronically into another application, the Enterprise Discharge Summary (EDS), which produces a comprehensive discharge summary for patients discharged from public hospitals in Queensland. The DMR and discharge summary can be transferred, via mail, e-mail or facsimile, to the patient's nominated general practitioner and community pharmacy, depending on the discharge process in place at the relevant hospital and technology compatibility with the applications used by the primary care providers. ${ }^{14,15}$ Studies exploring these initiatives have typically involved the hospitalcommunity interface in urban settings. ${ }^{3-6}$ There is little research on medication information transfer between community health services, between hospitals (rural and metropolitan) and rural community settings.

This study aimed to explore medication information transfer issues faced by rural healthcare providers and their perspectives on initiatives to optimise the process.

\section{METHOD}

This study was part of a broader research investigating medication supply and management issues in a rural community. ${ }^{16}$ Ethics approval to conduct the research was granted by The University of Queensland Behavioural and Social Sciences Ethical Review Committee, Griffith University Human Research Ethics Committee, University of Southern Queensland Human Research Ethics Committee and the human research ethics committee of the study health service district.

The study community comprised four towns in a rural health service district in Queensland, which cannot be named in compliance with the ethical requirement to preserve participant confidentiality. The average population across the four towns was 1500 , with a Pharmacy Access/Remoteness Index of Australia (PhARIA) categories 4 to $6 .{ }^{17}$ The PhARIA classifies pharmacies into 1 of 6 groups $(1=$ highly accessible to 6 
$=$ very remote). The availability of basic health services in these towns are outlined in Table 1.

Table 1. Health services available in the four Queensland towns

\begin{tabular}{ll}
\hline Towns & Health services \\
\hline Town 1 & $\begin{array}{l}1 \text { hospital, } 1 \text { medical practice, } 2 \text { single pharmacist } \\
\text { community pharmacies (1 with an intern pharmacist), } \\
1 \text { aged-care facility }\end{array}$ \\
Town 2 & $\begin{array}{l}1 \text { hospital, } 1 \text { medical practice, } 1 \text { single pharmacist } \\
\text { community pharmacy, } 2 \text { aged-care facilities (1 affiliated } \\
\text { with the hospital) }\end{array}$ \\
Town 3 & $\begin{array}{l}1 \text { hospital, } 1 \text { medical practice, } 1 \text { single pharmacist } \\
\text { community pharmacy (with an intern pharmacist), }\end{array}$ \\
& $\begin{array}{l}1 \text { aged-care facility } \\
\text { Town } 4\end{array}$ \\
$\begin{array}{l}1 \text { outpatients-only hospital, } 1 \text { medical practice (serviced } \\
\text { by a part-time locum doctor), } 1 \text { single pharmacist } \\
\text { community pharmacy, no aged-care facility }\end{array}$
\end{tabular}

A visiting accredited pharmacist provided Residential Medication Management Reviews in Towns 1, 2 and 3.

Identified health professionals were contacted via e-mail or telephone to ascertain their involvement in medication services (e.g. prescribing, administration, supply, provision of medication information). They were invited to participate in face-to-face interviews (45 minutes duration) during a 4-week period (September to October 2010). Interviews were conducted jointly by two of the study researchers using a semi-structured interview guide and were recorded with informed consent. Interview topics explored workforce issues, extended roles, training or support systems, service delivery methods and inter-professional relationships surrounding medication supply and management services in the study community. The topics were informed by a literature review and interviews with key informants external to the study community. ${ }^{16,18,19}$

On reaching theme saturation, interview data were manually transcribed and analysed thematically to enhance understanding and contextualise the findings otherwise not identified using electronic applications. The involvement of four researchers (2 data collectors, 2 project directors) added robustness to the interpretation of the themes.

\section{RESULTS}

Three doctors declined to be interviewed due to work pressures, while all of the other health professionals contacted accepted the invitation. Forty-nine health professionals from the local hospitals, mental health facilities, aged-care facilities and community health services (e.g. general practice, domiciliary nursing, community pharmacy) were interviewed. Their roles are outlined in Figure 1, while their age and years of service within the specified role and health facility are outlined in Table 2. Doctors in the study community $(\mathrm{n}=5)$ serviced the local hospital, general practice and agedcare facilities, a common practice to maximise the medical workforce across sectors (Figure 1).

While the interviewees discussed a range of topics, transcripts analysed thematically revealed concerns with communicating medication information, which resulted in difficulties in ensuring continuity of medication supply and optimal medication management. This concern was not identified as a key theme in Town 4 due to the lack of health facilities where medication information transfer processes could be investigated. Relevant findings are

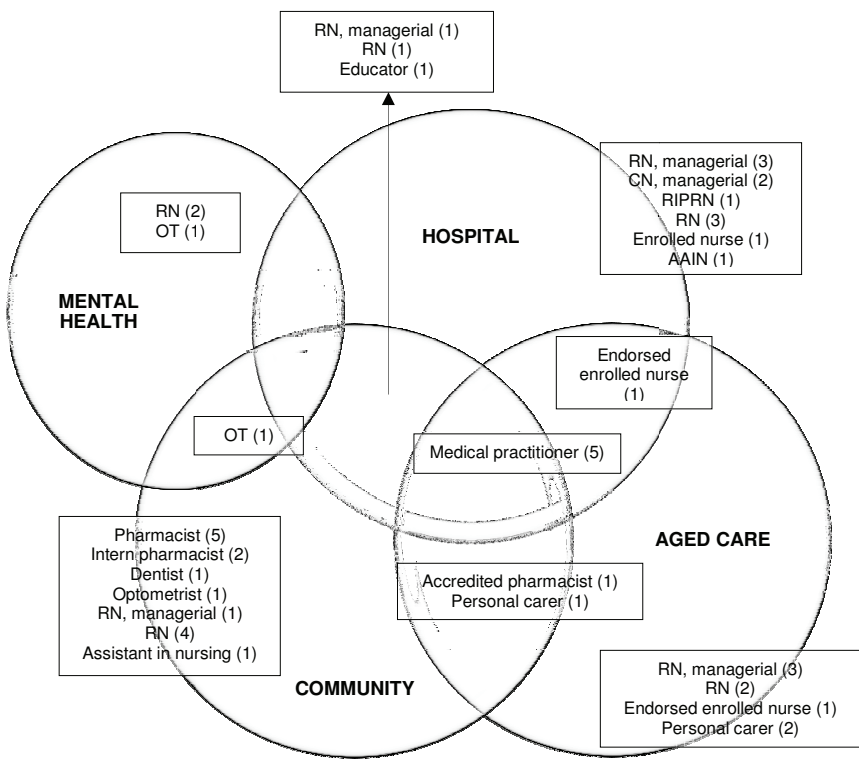

Figure 1. Participants' roles and health settings $(n=49) .{ }^{16}$ (AAIN = advanced assistant in nursing; CN = clinical nurse; OT = occupational therapist; RIPRN = rural and isolated practice-endorsed nurse; $\mathrm{RN}=$ registered nurse)

Table 2. Characteristics of participants

\begin{tabular}{lcc}
\hline Characteristics & No. of participants $(\mathbf{n}=\mathbf{4 9})$ \\
\hline Age (years) & $20-29$ & 4 \\
& $30-39$ & 13 \\
& $40-49$ & 12 \\
& $\geq 50$ & 20 \\
& $<0.5$ & 2 \\
Duration of & $\geq 0.5,<1$ & 7 \\
employment for the & $1-5$ & 15 \\
& $6-10$ & 11 \\
& $11-20$ & 9 \\
& $>20$ & 5 \\
\hline
\end{tabular}

reported descriptively, supported by de-identified quotations from participants.

\section{Within the Rural Community}

Despite assumptions that communication would be facilitated by the small size of the community, the shortage of resources challenged the healthcare providers to cope with medication-related processes. Nurses at aged-care facilities, community pharmacists and doctors reported difficulties in communicating medication information, such as prescription information for ongoing therapy, in a timely manner. This often occurred when there were incomplete or changes in doctors' orders/prescriptions, requiring changes to medication charts and/or dose administration aids. The resulting 'domino effect' increased the workload, with follow-up requests between the health professionals and delays in medication administration.

Participants commented on the lack of a coordinated system to reconcile medication information and to communicate medication or prescription information between the aged-care facility, community pharmacy and medical practice: 
I sat in [the doctor's] office with the pharmacist... All our drug charts were different. We had many discrepancies between what was on his computer, what was on our charts and what the pharmacy had. They are supposed to be all the same. (managerial aged care registered nurse [RN], HP27)

We get [prescription reminders] sent in from the pharmacist, aged-care facilities, community nurses, requesting repeat prescriptions... They're not always in sync with each other. It becomes difficult to keep track of prescriptions that have gone out... I end up going through and finding a lot of the duplications... It's very time-consuming. (doctor, HP30)

A community pharmacist and intern pharmacist extended their services to verify medication charts at the local aged-care facilities against pharmacy records and medications packed in dose administration aids. Although not as extensive as the Residential Medication Management Review provided by a visiting accredited pharmacist, this basic service enabled identification of medication-related errors, e.g. dosing errors, medication changes, drug-drug interactions.

We don't get paid for that... It's to weed out those problems that are happening... Making sure all our [dose administration aids] match the actual charts. (intern pharmacist, HP21)

One of the towns implemented an electronic record to facilitate medication and prescription information transfer between the aged-care facility and medical centre. Aged-care nursing staff and doctors in that town were supportive of such a system to enhance communication and thus increase awareness of patients' medication histories and current prescriptions. Health professionals in other towns cited confidentiality issues as the main barriers to implementing this type of system.

\section{Rural Hospital to other Health Services}

No hospital pharmacists were employed in the four study hospitals, and medication supply and basic pharmacy tasks were undertaken by the nurses. These nurses were reportedly trained by visiting hospital pharmacists to undertake medication reconciliation (admission and discharge) and to produce DMRs using the eLMS and EDS applications. Several nurses noted that when undertaking these tasks, they relied on assistance and verification from doctors. Feedback included increased workload and shortcomings in succession training, which led to unfamiliarity and inefficiency in using the technology.

It is 12 months [since we had EDS implemented]. [Hospital staff] had EDS training, but they've got by without needing it... They said, 'Why do we have to use it now?' The newer doctors [and staff] are using it, because they have probably been used to it in some other places and our old guys are picking it up as well. (hospital RN, HP20.2)

Usually the EDS [update] is done after the patient has gone home and it might be done the next morning... And it will be filed in their chart but the patient isn't actually given a copy of the EDS. (managerial hospital clinical nurse [CN], HP20.1)

Usually [the DMRs are] quite good, especially if a pharmacist has done them. When the nurses do them, they tend to not be very thorough... Some things might get missed. (community pharmacist, HP12)
A pharmacy-based educator noted that the doctors serviced both the hospital and community settings. Utilising the hospital applications to update patient medication information on top of their community-based applications was seen as duplication of workload.

The electronic discharge summary system that Queensland Health has doesn't talk directly well to [the GPs'] computer systems... In these towns, patients who go to hospitals are also [the doctors'] patients and so [the doctors] just take the medication history on their computer systems from the GP surgery. For their discharge summaries, [the doctors] think, 'Oh well, it's my patient.' It's just another computer system they've got to use. If they're already using their Medical Director at the medical centre, they prefer to just use one system. (pharmacy-based educator, HP45)

An example of when the hospital application was updated but not communicated to the patient and medical practice, was provided:

[An example of lady with diabetic foot ulcer who sees local GPs and has had frequent longer stays in the local hospital.] She was on [digoxin] and Lantus but was discharged with digoxin ceased and Lantus increased. Records at surgery were not updated so she returned to hospital on digoxin and reduced dose of Lantus. (hospital RN, HP20.2)

On balance, the majority of participants believed that these tools could achieve the target outcome of effective communication between health professionals.

That discharge summary is a fantastic idea. It is an extra bit of paperwork, though, for the health professionals in the [local] hospital. With the workload as it is, it's going to be very hard for [staff] to keep on top of that. The bigger hospitals have the advantage...they have more [staff] to do more of the clerical stuff. (doctor, HP30)

\section{Metropolitan Hospitals to Rural Health Services}

Several participants, including community and hospital nurses, were concerned about patients who were transferred from urban facilities to their rural residence. They provided examples where insufficient information, medications, support or care plans were provided to the patient and/or the primary care providers. This often complicated medication management and supply for ongoing therapy. These participants perceived that if the necessary medication information was discussed or communicated in an effective and timely manner prior to patients being discharged from the metropolitan hospital, the rural healthcare providers would be able to prepare for the patients' care and medications.

A managerial hospital nurse gave an example of a patient who experienced problems with continuity of her medication supply as a result of poor discharge planning. In this example, the local community pharmacist arranged temporary supply after difficulties obtaining the medication from regional hospitals, a problem that could have been averted with better pre-discharge communication to the rural healthcare providers.

We had a lady who went down to Brisbane... She had spinal injury and was put on gabapentin. She then came back, presented at the [local] hospital with [the script]... One, we don't stock gabapentin, two, we don't dispense [a repeat script]. We contacted the [nearest large hospital] and they said, 'We have a pharmacist, we can dispense, but we're not paying for it.' I contacted 
the [next largest hospital]... They went, 'No, no, no. It was prescribed at a hospital in Brisbane; it should be provided by them.' Meanwhile, 400-odd $\mathrm{km}$ away, that lady could have been without gabapentin for a couple of days, except we have a wonderful [community] pharmacist [who provided temporary supply]. [The original hospital that prescribed the medication eventually provided the full supply] (managerial hospital RN, HP25)

A managerial community nurse provided another example of the consequences of poor discharge planning from a metropolitan facility.

Discharging people on a Friday afternoon into a rural [town] isn't appropriate. A palliative care client was sent home on a Friday with no analgesia to get her through the weekend. She was sent home with enough for that day and that was it. Then the pharmacy didn't have them all. The biggest thing is making the [metropolitan facilities] aware of where we actually are. We are a town not close by. Our pharmacies, for safety reasons, don't keep large stocks of narcotics on the shelf, there is that delay in sorting out medications. (managerial community RN, HP39.1)

Nursing staff, community pharmacists and doctors commented on delays in relaying the necessary medication information (including the DMR) to the primary care providers and discrepancies in medication information, generating scepticism among healthcare providers about the reliability of the document.

With what's on the discharge medication summary and with what [the patients] are taking, there's often discrepancies... This often occurs with patients who have spent a substantial amount of time at larger facilities in metropolitan area with numerous changes to medications during that time, and also with patients who have had multiple discharges from tertiary and local hospitals... A lot can happen in a few weeks. (managerial community RN, HP39.1)

Participants, including nurses, also discussed the difficulties doctors have with post-discharge medication reconciliation and prescribing medications that were initiated by urban doctors without clear indications or action plans about the patient's medical conditions.

[Discharge planning] wasn't a focus in the acute setting. Patients were sent home with no scripts. It's Friday and the [local] doctors are booked out or they don't know the patient so they won't write a script... You can't blame [the local doctors]. (community RN, HP39.2)

Sometimes the DMRs are pretty vague. Sometimes the patients don't have the DMR. Sometimes [the patient] brings a little brown packet that has 5 tablets in it, with no name on it. Sometimes the labels are not very clear. Maybe that's not happening so much now, but sometimes you have trouble to work out what they are. (doctor, HP35)

A managerial hospital clinical nurse added that all hospitals should provide sufficient quantities of the discharge medications for ongoing therapy, and this should be implemented consistently.

It is good that [patients] come back [to the town] with [at least] 7-day supply, because they often transfer back in the evening or on a weekend... It's often very daunting when [patients] come back with a list of drugs and we don't have half of them... You have to go somewhere else to get what they need. (managerial hospital CN, HP20.1)
The managerial community nurse (HP39.1), on the other hand, recommended that a step-down protocol should be adopted, with rural patients being transferred from urban facilities to the local hospital, before being discharged home. This would require communication of medication-related information to the rural hospital, and enable the local health professionals to determine the patient's coping mechanisms and medication needs to ease the patient's transition to their home on discharge.

\section{DISCUSSION}

Although our findings may not be specific to rural communities, the issues relating to poor communication of medication-related information are significant in rural areas where there is less capacity for health professionals to resolve medication-related issues. This qualitative study has provided information from the perspectives of rural healthcare providers at the 'coal face', to inform stakeholders of the issues and improvements that can be made to medication information transfer processes.

The study identified inefficiencies in the existing patient information transfer system, which challenged the provision of continuous health care and medication support to patients. The following key issues were identified:

- discrepancies in medication records and lack of a coordinated system to communicate medication or prescription information in a timely manner within the rural community;

- challenges for rural hospital staff to undertake medication reconciliation and information transfer tasks; and

- inadequate communication of medication-related information between urban hospitals and rural healthcare providers to ensure continuity of medication supply and ongoing management.

There is potential to implement a medication information liaison model to address these inefficiencies. ${ }^{6,8,13}$ The Pharmaceutical Society of Australia's Professional Practice Standards outline the scope of a liaison pharmacist, with roles incorporating medication review and reconciliation..$^{20}$ This model would be led by a community pharmacist in rural regions where there is an absence of hospital pharmacists, given that a patient's medication record at the local pharmacy is likely to be the most current and accurate available. ${ }^{3,11}$ It is anticipated that such a model will reduce the workload of other healthcare providers, allowing them to focus on their key professional responsibilities. It could also facilitate communication between health professionals in secondary/tertiary facilities and rural community healthcare providers. ${ }^{2,6}$ Implementation could be limited by workforce issues, remuneration, confidentiality and access to medication records at the medical centre or hospital. ${ }^{11,13}$

The use of electronic applications to enhance medication information communication has been explored. Queensland Health's state-wide eLMS and EDS applications were designed to facilitate processes for healthcare providers, thereby improving medication record keeping and medication information transfer to the community. ${ }^{14,15}$ However, as these applications are only available in public hospitals, community healthcare providers were unable to access these for patient updates. Doctors reported duplication of workload when 
using separate applications when servicing the hospital and medical practice. Healthcare providers also reported inefficiencies such as delays in documentation, inaccurate DMRs and DMRs generated from multiple discharges. While the potential for electronic applications is user-dependent and can be impacted by time factors, system incompatibility or inaccessibility, workload and technological unfamiliarity, studies have demonstrated their benefits in facilitating medication-related processes..$^{6,14,15}$ Further research is warranted to investigate the reported shortcomings to support and improve the use of such systems.

The use of a single medication record to promote consistency and to ease medication information transfer has been explored in one of the study towns. A universal communication system across health settings is ideal to promote continuity of medication therapy and to address issues highlighted in the study. ${ }^{5}$ A national electronic health record with optional participation that is supported by the national broadband communications network in rural areas, has been mooted for some time. It is anticipated that such a system will significantly improve patient information transfer nationwide. ${ }^{6,21,22}$

Health professionals reported poor communication between the hospital and community providers and inefficiencies in using the electronic applications to facilitate medication information transfer. These issues complicated continuity of medication management for discharged patients returning to their rural residence from urban facilities with nil-to-minimal medication information or supply, deviating from the continuity of care principles outlined by the Australian Pharmaceutical Advisory Council. ${ }^{2}$ Prior to the introduction of the PBS reforms in Queensland Health hospitals, on discharge the hospitals generally provided patients with a 7-day supply of medications. With the PBS hospital reforms, patients are being issued PBS prescriptions on discharge to be dispensed by the hospital or community pharmacy.,11 However, our study revealed continuity of medication supply was an issue for patients discharged after-hours or on weekends, where pharmacy services were scarce or certain medications unavailable in rural areas. Participants' suggestions to address this issue included improving and communicating discharge plans to promote continuity of medication management for ongoing therapy for discharged patients.

Our study was not designed to compare rural and urban settings, but to identify issues in the existing system relevant to medication supply and management processes in the study community. The focus was the general processes involved in medication information transfer rather than to evaluate the quality of information provided or transferred by healthcare providers or electronic applications. Several participants were reluctant to reveal issues that may relate to other healthcare providers and feared judgment of their practice. However, with the assurance of anonymity, they gave examples of authentic practice and commented meaningfully on solutions or the initiatives already in place.

In conclusion, key issues were identified relating to medication information transfer between healthcare providers and between healthcare settings, which impacted on optimal medication management for rural patients. Strategies to address these issues could include a medication liaison model for the local community pharmacist; a review of the implementation and use of electronic applications; and improvement in discharge protocols and network/communication between rural healthcare providers and secondary/tertiary facilities.

Competing interests: None declared

\section{References}

1. Australian Council for Safety and Quality in Health Care. Second national report on patient safety: improving medication safety. Canberra: The Council; 2002 .

2. Australian Pharmaceutical Advisory Council. Guiding principles to achieve continuity in medication management. Canberra: Commonwealth of Australia; 2005 .

3. Couch G, Quah SM, Ng YC, McKellar A. Continuity of medication supply and provision of patient information on discharge: development of a survey tool. J Pharm Pract Res 2007; 37: 210-13

4. O'Leary KM, Allinson YM, Stowasser DA, Dowling H. Survey of implementation levels and pharmacy requirements for the continuum of quality use of medicines between hospital and community. J Pharm Pract Res 2003; 33 : 93-100.

5. Pierce D, Fraser G. An investigation of medication information transfer and application in aged care facilities in an Australian rural setting. Rural Remote Health 2009; 9: 1090.

6. Semple SJ, Roughead E. Medication safety in acute care in Australia: where are we now? Part 2: a review of strategies and activities for improving medication safety 2002-2008. Aust N Z Health Policy 2009; 6: 24.

7. Easton K, Morgan T, Williamson M. Medication safety in the community: a review of the literature. Sydney: National Prescribing Service; 2009.

8. Stowasser D, Collins DM, Stowasser M. A randomised controlled trial of medication liaison services - patient outcomes. J Pharm Pract Res 2002; 32: 133-40.

9. Carroll M, Galbraith KJ, Dooley MJ. Potential support roles for pharmacy technicians in provision of clinical pharmacy services. J Pharm Pract Res 2003; 33: 186-93.

10. Fogarty G, McKeon CM. Patient safety during medication administration: the influence of organizational and individual variables on unsafe work practices and medication errors. Ergonomics 2006; 49: 444-56.

11. Jestrimski $\mathrm{K}$. Management challenges in large rural hospital pharmacy departments. Aust J Hosp Pharm 1999; 29: 202-5.

12. Burridge N. SHPA standards of practice for the provision of medication reconciliation. J Pharm Pract Res 2007; 37: 231-3.

13. Vuong T, Marriott JL. Potential role of the community liaison pharmacist: stakeholder views. Int J Pharm Pract 2006; 14: 135-48.

14. Arkinstall R, Lum E, Reid C, Maclean C. Supporting rural nurses in safe and appropriate medication management. Woolloongabba: Association of Queensland Nurse Leaders; 2007. Available from <www.aqnl.org.au/ default.asp?PageID $=79 \& n=2007+$ Conference + Speakers + Papers $>$.

15. McKay J. Streamlining medication management into the community. Brisbane: Queensland Health; 2007. Available from <www.crepatientsafety.org.au/ seminars/clinical-handover/jennie-Mckay.pdf>.

16. Hattingh L, Emmerton L, Tan A, Jarvis V. An exploratory study of extended health care practitioner roles in medication supply and management in a rural community: final report. Brisbane: The University of Queensland, Griffith University; 2011. Available from $</$ espace.library.curtin.edu.au: $80 / \mathrm{R}$ ? func $=\mathrm{d}$ bin-jump-full\&local base $=$ gen01-era02\&object $\mathrm{id}=165686>$.

17. GISCA. Pharmacy ARIA - PhARIA. Adelaide: The University of Adelaide; 2010. Available from <www.gisca.adelaide.edu.au/projects/pharia.html $>$. 18. Tan A, Emmerton L, Hattingh L, Jarvis V. Medication supply and management in rural Queensland: views of key informants in health service provision. Res Social Adm Pharm. In press.

19. Tan A, Emmerton L, Hattingh L. A review of the medication pathway in rural Queensland, Australia. Int J Pharm Pract. In press.

20. Pharmaceutical Society of Australia. Professional practice standards: version 4. Deakin: PSA; 2010

21. National Health and Hospitals Reform Commission. A healthier future for all Australians: final report. Barton: Commonwealth of Australia; 2009.

22. Miller S. Rural and remote health workforce innovation and reform strategy: draft background paper. Adelaide: Health Workforce Australia; 2011. Available from < www.hwa.gov.au/work-programs/workforce-innovation-and-reform/ rural-and-remote-health-workforce>.

\section{Received: 1 November 2011}

Revisions requested after external review: 30 December 2011

Revised version received: 3 February 2012

Accepted: 6 February 2012

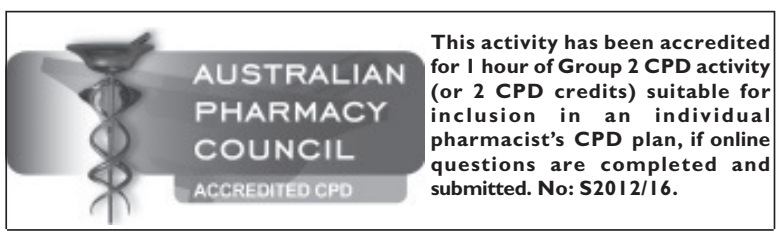

